ARTICLE

DOI: $10.1038 /$ s41467-018-07670-4

\title{
An AlEgen-based 3D covalent organic framework for white light-emitting diodes
}

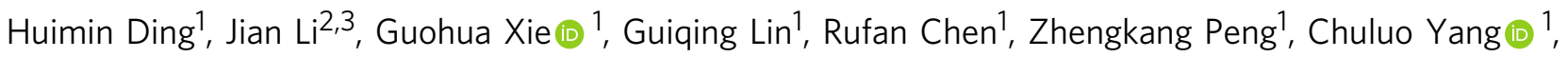
Baoshan Wang (1) ${ }^{1}$, Junliang Sun (1) ${ }^{2,3} \&$ Cheng Wang ${ }^{1}$

The design and synthesis of three-dimensional covalent organic frameworks (3D COFs) have still been considered as a big challenge. Here we report the design and synthesis of an AlEgen-based 3D COF (3D-TPE-COF), with a high surface area $\left(1084 \mathrm{~m}^{2} \mathrm{~g}^{-1}\right)$. According to powder $X$-ray diffraction and continuous rotation electron diffraction analyses, 3D-TPE-COF is identified to adopt a seven-fold interpenetrated pts topology. Interestingly, 3D-TPE-COF emits yellow fluorescence upon excitation, with a photoluminescence quantum yield of $20 \%$. Moreover, by simply coating 3D-TPE-COF onto a commercial blue light-emitting diode (LED), a prototype white LED (WLED) under continuously driving without degradation for $1200 \mathrm{~h}$ was demonstrated. The present work suggests the possibility of using COF materials for stable WLEDs, which will greatly inspire us to design and synthesize fluorescent 3D COFs and facilitate the development of COF-based WLEDs in future.

\footnotetext{
${ }^{1}$ Key Laboratory of Biomedical Polymers (Ministry of Education), College of Chemistry and Molecular Sciences, Wuhan University, Wuhan 430072, China.

${ }^{2}$ College of Chemistry and Molecular Engineering, Beijing National Laboratory for Molecular Sciences, Peking University, Beijing 100871, China. ${ }^{3}$ Department of Materials and Environmental Chemistry, Stockholm University, Stockholm 10691, Sweden. These authors contributed equally: Huimin Ding, Jian Li, Guohua Xie. Correspondence and requests for materials should be addressed to J.S. (email: junliang.sun@pku.edu.cn) or to C.W. (email: chengwang@whu.edu.cn)
} 
C ovalent organic frameworks (COFs) represent an emerging class of crystalline porous polymers with periodic two- or three-dimensional (2D or 3D) structure ${ }^{1-4}$. Due to their permanent porosity, high stability, and designable functionality, COFs have gained considerable attention as promising applications in gas storage and separation ${ }^{5,6}$, catalysis ${ }^{7-10}$, sensing $^{11-13}$, optoelectronics ${ }^{14-17}$, energy storage ${ }^{18-21}$, etc. For all these reported systems, most of the examples are focusing on $2 \mathrm{D}$ COFs, and their synthesis and characterization have been relatively well established ${ }^{22-27}$. By contrast, since Yaghi and coworkers reported the first example in $2007^{28}$, only a few 3D COFs have been announced over the past decade ${ }^{29-39}$, mainly due to their limited availability of molecular building blocks and synthetic challenge. However, it should be emphasized here, as these $3 \mathrm{D}$ architectures can allow hierarchical arrangement of nanopores and possess numerous open sites, some reported 3D COFs have shown interesting applications, for example, size-selective catalysis $^{36}$. Considering their unique structures and properties, the rational design and synthesis of 3D COFs with specific functions are highly demanded.

Aggregation-induced emission (AIE) is a novel photophysical phenomenon in which the luminogens are nonemissive in dilute solution but become highly emissive in aggregate state ${ }^{40,41}$. Since firstly coined by Tang and coworkers in $2001^{42}$, AIE luminogens (AIEgens) based materials have gained intensive attentions and shown broad applications in light-harvest ${ }^{43}$, light-emission ${ }^{44}$, chemo- and biosensors $^{45,46}$, bioimaging ${ }^{47}$, and so on. The construction of AIEgen-based 3D COFs, which will precisely integrate AIEgens into $3 \mathrm{D}$ porous crystalline organic framework, should be very interesting ${ }^{48,49}$. First, the rigid skeleton can restrict the intramolecular rotation, vibration, and motions of AIEgens. Consequently, the obtained 3D COFs may be fluorescent, which can provide a unique platform for us to understand the underlying mechanism of AIE. Second, as solid-state fluorescent porous materials, these $3 \mathrm{D}$ COFs may find interesting applications in different areas, for example, sensing and optoelectronic devices. However, due to the synthetic challenge, AIEgen-based 3D COFs have not been reported yet.

Recently, we reported the design and synthesis of 3D COFs bearing pyrene ${ }^{29}$ or porphyrin ${ }^{30}$ units, starting from tetrahedral and quadrilateral building blocks connected through $[4+4]$ imine condensation reactions. According to these results, we believe it is reasonable to construct other $3 \mathrm{D}$ COFs via this topology design. Therefore, we decided to choose the representative AIEgens, tetraphenylethylene (TPE) ${ }^{41}$, as the quadrilateral core to synthesize AIEgen-based 3D COFs.

In this work, we reported the design and synthesis of a TPEbased 3D COF, named 3D-TPE-COF (Fig. 1). Our result clearly shows that the $3 \mathrm{D}$-TPE-COF is a microporous material with high surface area, and by using the newly developed continuous rotation electron diffraction (cRED) method, 3D-TPE-COF is identified to adopt a sevenfold interpenetrated pts (the net of the platinum and sulfur atoms in $\mathrm{PtS}$ ) topology with $\mathrm{P} 2 / \mathrm{c}$ space group. Interestingly, 3D-TPE-COF emits yellow fluorescence upon excitation, with a photoluminescence quantum yield (PLQY) of $20 \%$. Moreover, by simply coating 3D-TPE-COF onto a commercial blue light-emitting diode (LED), a prototype white LED (WLED) was fabricated. The Commission International de I'Eclairage (CIE) coordinates of COF-based WLED were determined to be $(0.30,0.35)$, which are close to that of the pure white light. In addition, this WLED exhibited no degradation after aging for $1200 \mathrm{~h}$ at ambient condition.

\section{Results}

Synthesis and characterization of 3D-TPE-COF. 3D-TPE-COF was synthesized by condensation of the reported tetra $(p$-aminophenyl)methane (TAPM) ${ }^{50}$ and 1,1,2,2-tetrakis(4-formyl-(1,1'biphenyl))ethene (TPE-Ph-CHO $)^{51}$ in a mixture of $o$-dichlorobenzene, mesitylene and $6 \mathrm{M}$ aqueous acetic acid (10: 10: 3, v/v/ v) at $120^{\circ} \mathrm{C}$ for 7 days (Fig. 1). After exhaustively washed by Soxhlet extraction with tetrahydrofuran, acetone, and dichloromethane, 3D-TPE-COF was isolated in $85 \%$ yield as a yellow powder insoluble in common organic solvents and water. From the Fourier transform infrared (FT-IR) spectrum, 3D-TPE-COF exhibited the diagnostic vibrational peak of the imine bond at $1625 \mathrm{~cm}^{-1}$ (Supplementary Figure 2), which is the same as that of the model compound (Supplementary Figure 1). In addition, the ${ }^{13} \mathrm{C}$ cross-polarization with total suppression of sidebands (CPTOSS) NMR spectrum of 3D-TPE-COF also confirmed the formation of the expected $\mathrm{C}=\mathrm{N}$ bond at $158.3 \mathrm{ppm}$ (Supplementary Figure 3). Furthermore, field-emission scanning electron microscopy images showed that 3D-TPE-COF has a rod-like morphology (Supplementary Figure 4), consisting of microcrystals.

The powder X-ray diffraction (PXRD) experiment was performed to demonstrate the crystalline nature of 3D-TPECOF. As shown in Fig. 2, the experimental PXRD pattern displayed amounts of intense diffraction peaks, indicating the presence of a long-range ordered structure. However, it was very difficult to determinate the structure from PXRD data, due to the serious overlap of diffraction peaks. Since the nano- and micrometer-sized crystals can be treated as single crystal in electron crystallography, RED technique has recently been applied as a powerful tool for the structure determination of 3D COFs with dia topology ${ }^{31-33}$. The newly developed technique, $\mathrm{cRED}^{52}$, was then used to collect 3D electron diffraction, in which the time for data collection can be reduced to less than 5 min (see Supplementary method 3 for details). After carefully analysing the cRED data, we successfully located the central carbon atoms of tetrahedral and quadrilateral building blocks (Supplementary Figure 8) by SHELXT ${ }^{53}$. Based on the atom coordinates determined from cRED data, we were able to build a structure model with seven-fold interpenetrated pts net (Fig. 3) using the Materials studio software package, in which the calculated PXRD patterns of optimized model matched well with the experimental data (Fig. 2 and Supplementary Figure 9). The Le Bail fitting was performed on the experimental PXRD of 3D-TPE-COF, which yields a unit cell with parameters $(a=27.331(1) \AA$, $b=8.543(5)$ $\AA, c=31.508(0) \AA, \alpha=\gamma=90^{\circ}$, and $\left.\beta=90.476(0)^{\circ}, P 2 / c\right)$. The final $R_{\mathrm{wp}}$ and $R_{\mathrm{p}}$ values converged to 3.59 and $2.38 \%$, respectively. Therefore, $3 \mathrm{D}$-TPE-COF is identified to adopt a seven-fold interpenetrated pts topology with $P 2 / c$ space group.

The permanent porosity of 3D-TPE-COF was determined by $\mathrm{N}_{2}$ adsorption measurement at $77 \mathrm{~K}$. As shown in Fig. 4a, 3DTPE-COF showed a type I isotherm, which is characteristic of microporous materials. The Brunauer-Emmett-Teller (BET) surface area and total pore volume were calculated to be 1084 $\mathrm{m}^{2} \mathrm{~g}^{-1}$ and $0.55 \mathrm{~cm}^{3} \mathrm{~g}^{-1}$, respectively. It is worth noting that this BET surface area is much higher than that of its amorphous analog $\left(512 \mathrm{~m}^{2} \mathrm{~g}^{-1}\right)^{51}$, which is also synthesized from TAPM and TPE-Ph-CHO. The pore width distribution calculated by the quenched solid density functional theory (QSDFT) model revealed one major peak centered at $0.57 \mathrm{~nm}$ (Fig. $4 \mathrm{a}$ ). We also studied the $\mathrm{CO}_{2}$ adsorption behavior of 3D-TPE-COF (Fig. 4b), and the uptake value was $72 \mathrm{~cm}^{3} \mathrm{~g}^{-1}$ at $273 \mathrm{~K}$ and $45 \mathrm{~cm}^{3} \mathrm{~g}^{-1}$ at $298 \mathrm{~K}$. In addition, the thermogravimetric analysis revealed that 3D-TPE-COF starts to decompose at $400^{\circ} \mathrm{C}$ (see Supplementary Figure 6), indicating the high thermal stability. More importantly, this COF showed good chemical stability (Fig. 4c). After dispersing 3D-TPE-COF in common organic solvents, water 


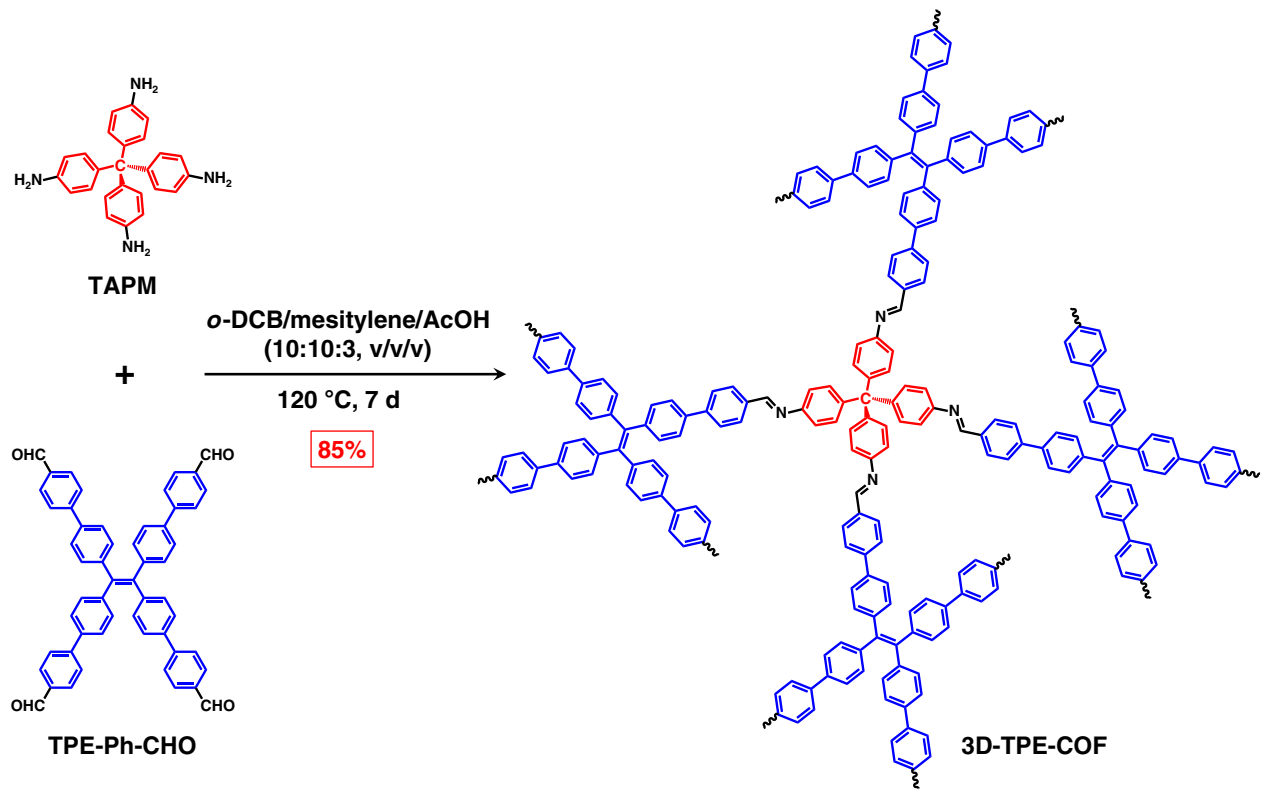

Fig. 1 Chemical Structure. Schematic representation of the synthesis of 3D-TPE-COF

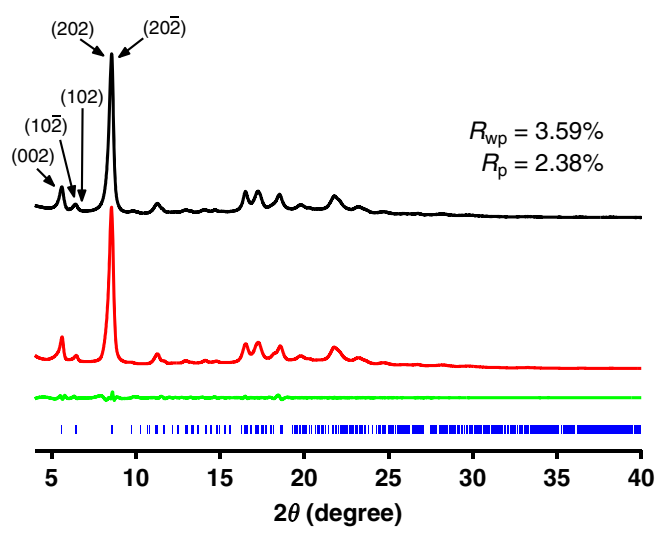

Fig. 2 Powder X-ray diffraction patterns of 3D-TPE-COF. PXRD profiles of experimental pattern (black curve), Le Bail fitting pattern (red curve), their difference (green curve) and Bragg position from the seven-fold interpenetrated pts structure (blue curve)

and alkaline solution for $24 \mathrm{~h}$, the COF samples still exhibited strong diffraction peaks in PXRD patterns. This is different from the reported boronate-linked 2D TPE-Ph $\mathrm{COF}^{12}$, which will lose crystalline structure upon exposure to water.

Considering the unique photophysical phenomenon of TPE chromophore, we then investigated the fluorescent properties of 3D-TPE-COF. Before that, we studied the fluorescence spectrum of the model compound in solid state. As shown in Supplementary Figure 12, the powder of the model compound exhibited yellow-green emission peaking at $534 \mathrm{~nm}$ upon excitation. However, the PLQY of the model compound is considerably low (6.6\%), as the photo-induced electron transfer from TPE units to imine groups will quench the fluorescence ${ }^{54}$. Interestingly, 3D-TPE-COF can emit yellow light with an emission maximum at $543 \mathrm{~nm}$ (Fig. 4d), but with a much higher PLQY (20\%). According to the crystal structure (Fig. 3d), we believe this enhancement can be ascribed to the aggregation of TPE units in the 3D framework. In addition, the rigid skeleton can restrict the relaxation of TPE units, which may also contribute this enhancement. Due to the fluorescent and porous nature of 3DTPE-COF, we tested its chemosensing behavior by choosing the commercially available picric acid (PA) as explosive model (Supplementary Figure 14) in water. Obviously, the fluorescence of 3D-TPE-COF was quenched when PA was gradually added into the suspension, and the Stern-Volmer curve quenching constant $\left(K_{\mathrm{sv}}\right)$ was estimated to be $3.3 \times 10^{4} \mathrm{M}^{-1}$.

Utilizing 3D-TPE-COF as coating material for WLED. WLEDs have attracted extensive attention due to their wide applications in display and lighting systems ${ }^{55-58}$. A common approach to fabricate WLEDs is coating a yellow-emitting phosphor on a blue LED chip, and the white light can be produced from the combined down-converted emissions of the phosphor and the blue component of the LED chip. As 3D-TPE-COF emits yellow fluorescence upon excitation with blue light, we then explore the possibility of using this 3D COF for WLEDs. By using a simple dip-coating procedure (see Supplementary Method 6 for details), a thin film of 3D-TPE-COF can be homogeneously coated onto the surface of a commercially available blue LED lamp (peaking around $450 \mathrm{~nm}$ ). As shown in Fig. 5, when the LED was turned on, bright white light was generated. The CIE coordinates were determined to be $(0.30,0.35)$, which are close to the standard coordinates for pure white light $(0.33,0.33)$. To explore the potential of practical application, we further tested the stability of this prototype WLED (Fig. 5b). Obviously, the luminescence of the COF-coated WLED is considerably stable under continuously driving at ambient condition for $1200 \mathrm{~h}$. Such highly stable WLED has seldom been reported for the one with a downconversion layer of organic compounds. Therefore, by simply coating onto the commercial blue chips, 3D-TPE-COF has shown interesting application in robust, low-cost, and rare-earth metalfree WLEDs.

\section{Discussion}

We have reported the design and synthesis of an AIEgen-based 3D COF, 3D-TPE-COF, starting from tetrahedral and quadrilateral building blocks connected through $[4+4]$ imine condensation reactions. The obtained 3D-TPE-COF was characterized by several techniques, and according to cRED analyses, it is identified to adopt a seven-fold interpenetrated pts topology with $P 2 / c$ space group. Interestingly, the immobilization 
a

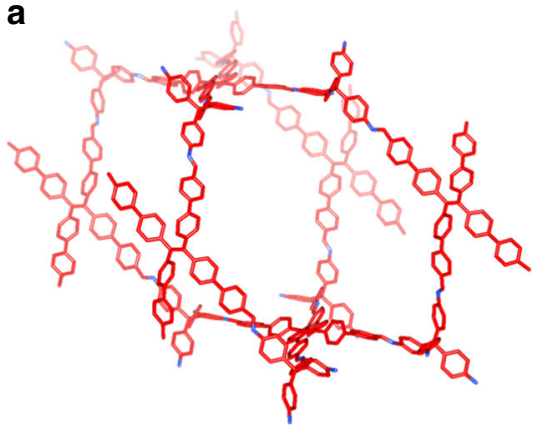

b

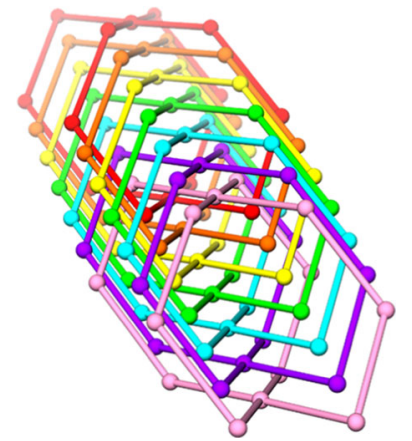

d

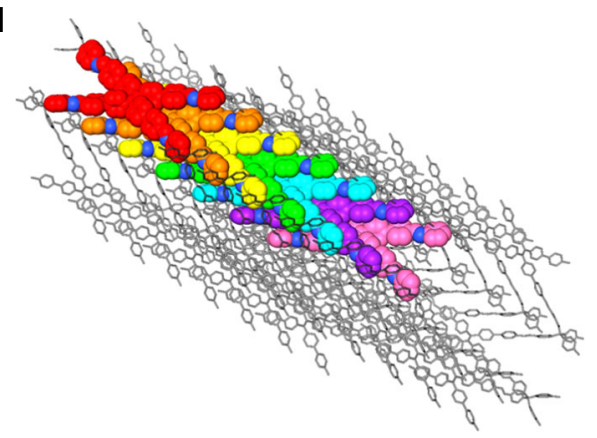

C

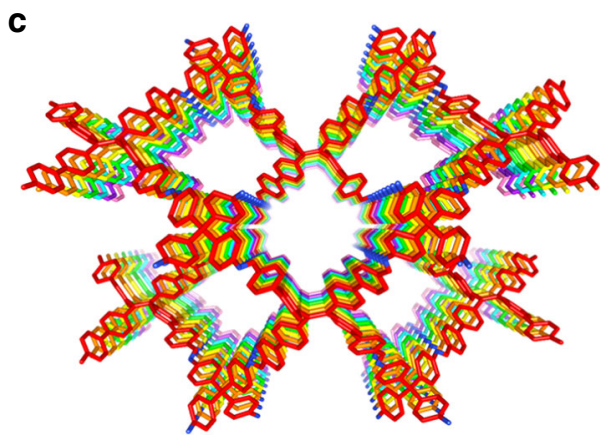

Fig. 3 Structural representations of 3D-TPE-COFs. a Single pts network; $\mathbf{b}$ seven-fold interpenetrated pts topology; $\mathbf{c}$ the porous structure of 3D-TPE-COF; d the stacking behavior of TPE molecules in framework. Hydrogen atoms are omitted

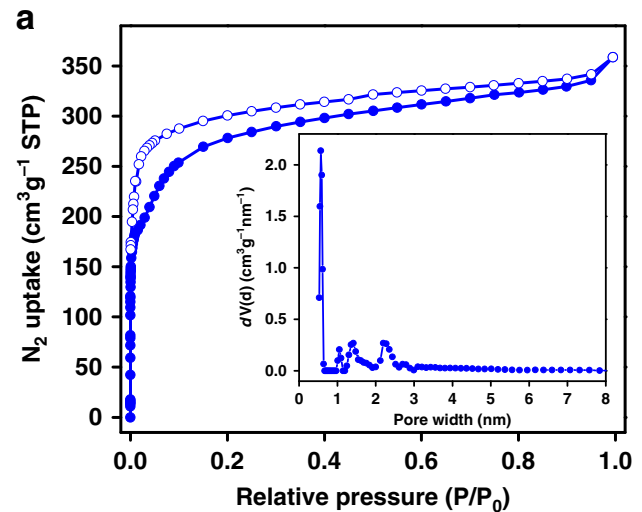

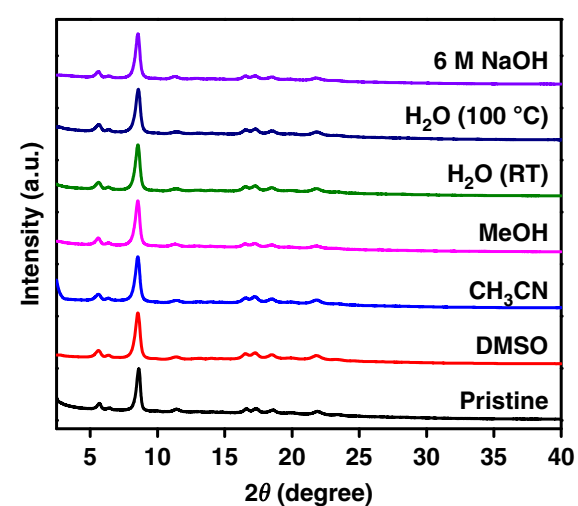
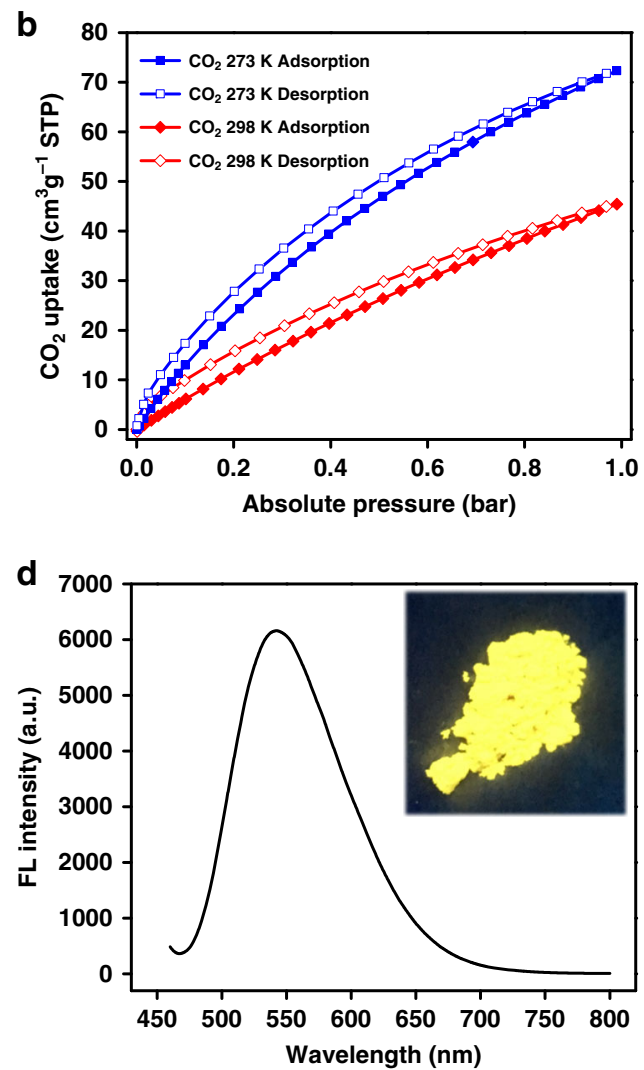

Fig. 4 Characterization of 3D-TPE-COF. a $\mathrm{N}_{2}$ adsorption and desorption isotherms of 3D-TPE-COF at 77 K. The inset is the pore size distribution of 3DTPE-COF calculated from the quenched solid density functional theory; $\mathbf{b} \mathrm{CO}_{2}$ adsorption and desorption isotherms of 3D-TPE-COF at $273 \mathrm{~K}$ and $298 \mathrm{~K}$; c PXRD patterns of 3D-TPE-COF after treatment in different solvents for $24 \mathrm{~h}$; $\mathbf{d}$ solid-state fluorescence spectrum of $3 \mathrm{D}-\mathrm{TPE}-\mathrm{COF}\left(\lambda_{\mathrm{ex}}=450 \mathrm{~nm}\right)$. The inset is the photograph of 3D-TPE-COF powders under UV light irradiation 

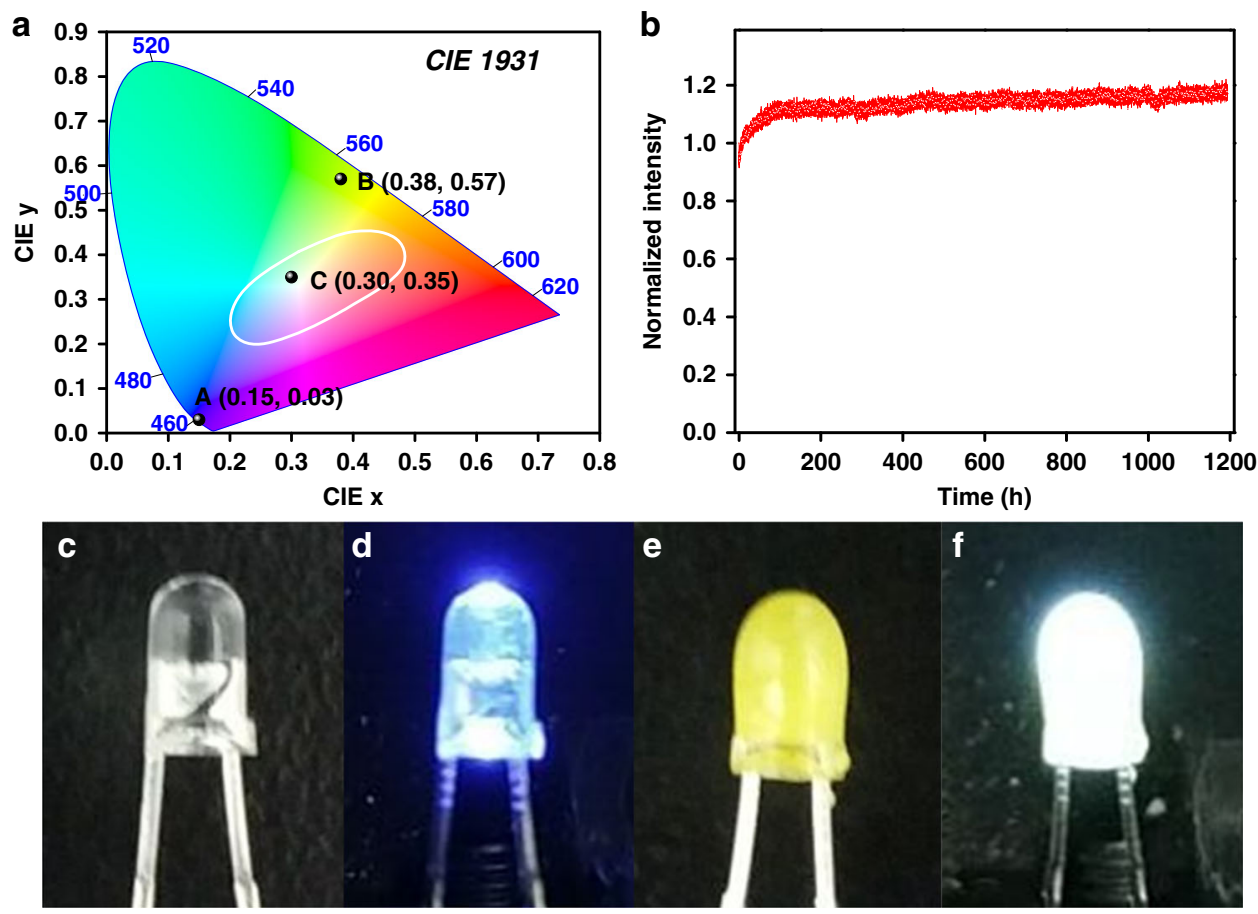

Fig. 5 Characterization and photographs of the LEDs. a CIE-1931 chromaticity diagram and the positions (marked by the dots) for blue LED (0.15, 0.03; a $3 D-T P E-C O F\left(0.38,0.57 ; \mathbf{b}\left(\lambda_{\text {ex }}=450 \mathrm{~nm}\right)\right.$, and COF-coated LED $(0.30,0.35 ;(\mathbf{c})$. b Normalized luminescence intensity vs. time curve of the 3D-TPE-COFcoated WLED driven at $2 \mathrm{~mA}$. Photographs of the LEDs: $\mathbf{c}$ a reference blue LED (not turned on), $\mathbf{d}$ the reference blue LED turned on (emission with blue light), e the same LED coated with a thin layer of 3D-TPE-COF (not turned on), and $\mathbf{f}$ the coated LED turned on (emission with white light)

of TPE into 3D COF can allow the resulting 3D-TPE-COF emit yellow fluorescence upon excitation. Moreover, by simply coating 3D-TPE-COF onto a commercial blue LED, the COF-based WLED was constructed with the CIE coordinates of $(0.30,0.35)$, which showed no degradation under continuously driving at ambient condition for $1200 \mathrm{~h}$. The present work demonstrates that fluorescent 3D COFs is feasible to fabricate rare-earth metalfree WLEDs, which will inspire us to facilitate the development of other fluorescent 3D COFs with higher quantum yields. In addition, considering the intrinsic porosity, the accommodation of dye molecules within the pores of fluorescent 3D COFs may also offer another strategy to construct WLEDs. Currently, these studies are undergoing in our lab.

\section{Methods}

Materials. All the reagents and starting materials were purchased from Acros, Adamas or TCI, and used without further purification unless otherwise noted. Dehydrated solvents were obtained after treating solvents with standard procedures. Tetrakis-(4-aminophenyl)methane (TAPM) $)^{50}$ and 1,1,2,2-tetrakis(4-formyl$\left(1,1^{\prime}\right.$-biphenyl) $)$ ethene (TPE-Ph-CHO $)^{51}$ were synthesized according to the literature. The blue LED [https://item.jd.com/12630843985.html] was purchased online.

Characterizations. High-resolution mass spectra were collected on DIONEX UltiMate 3000 \& Bruker Compact TOF and Bruker Solarix mass spectrometers. Solid-state NMR spectra were recorded at ambient temperature on a Bruker AVANCE III 400M spectrometer. Fourier transform infrared (FT-IR) spectra were recorded on a Nicolet iN10 micro FTIR Spectrometer. Elemental analysis was conducted on a Flash EA 1112. The PXRD patterns were obtained on a Rigaku Smartlab X-ray diffractometer with $\mathrm{Cu} \mathrm{K} \alpha$ line $(\lambda=1.54056 \AA)$. Thermogravimetric analysis was performed on a TGA-Q500 from 30 to $800^{\circ} \mathrm{C}$ at the rate of $10^{\circ}$ $\mathrm{C} \mathrm{min}^{-1}$ without equilibration delay. Field-emission scanning electron microscopy (FE-SEM) images were performed on a Zeiss IIGMA operating at an accelerating voltage ranging from 0.1 to $20 \mathrm{kV}$. The samples were prepared by dispersing the material onto conductive adhesive tapes attached to a flat aluminum sample holder and then coated with gold. Nitrogen isotherms were measured at $77 \mathrm{~K}$ using an Autosorb-iQ (Quantachrome) surface area size analyzer.
The 3D electron diffraction data were collected on a $200 \mathrm{kV}$ JEOL JEM-2100 transmission electron microscope, which was equipped with a quad hybrid pixel detector (Timepix). For data collection, a micro single crystal was selected and placed in the electron beam, and then the $\mathrm{Z}$ height was adjusted to the mechanical eucentric height. The selected-area ED patterns were captured from the crystal continuously when the goniometer was rotated. Finally, 374 ED patterns were recorded and the tilt rang was from $-52.29^{\circ}$ to $34.3^{\circ}$ with the tilt step of $0.23^{\circ}$. The total time for data collection was $191 \mathrm{~s}$. UV-Vis spectra were recorded on a SHIMADZU UV-3600 UV-vis-NIR spectrophotometer. Fluorescence spectra were recorded on a HITACHI F-4600 spectrofluorometer. Absolute quantum yields were obtained using a Quantaurus-QY measurement system (C9920-02, Hamamatsu Photonics) and all the samples were excited at $450 \mathrm{~nm}$. A PR735 SpectraScan Spectroradiometer (Photo Research) was used to simultaneously record the electroluminescent spectra and CIE coordinates. The intensity vs. time curve of the COF-coated LED driven at $4 \mathrm{~mA}$ was recorded by a lifetime measurement system (Crysco) at room temperature.

Synthesis of 3D-TPE-COF. A Pyrex tube was charged with TAPM (25.5 mg, 0.067 $\mathrm{mmol}$ ), TPE-Ph-CHO (50 mg, $0.067 \mathrm{mmol}$ ), $2.0 \mathrm{~mL}$-dichlorobenzene and $2.0 \mathrm{~mL}$ mesitylene. The mixture was sonicated for $10 \mathrm{~min}$, followed by slow addition of 0.6 $\mathrm{mL}$ of $6 \mathrm{M}$ aqueous acetic acid. After that, the tube was degassed by three freeze - pump-thaw cycles, sealed under vacuum and then heated at $120^{\circ} \mathrm{C}$ for $7 \mathrm{~d}$. The reaction mixture was cooled to room temperature and the resulting precipitate was filtered off, exhaustively washed by Soxhlet extractions with tetrahydrofuran, acetone, and dichloromethane for $24 \mathrm{~h}$. The resulting yellow powder was dried at $80^{\circ} \mathrm{C}$ under vacuum for overnight to give $3 \mathrm{D}$-TPE-COF in $85 \%$ yield. Elemental analysis for the calculated: C, $89.74 \%$; H, 4.96\%; N, 5.30\%. Found: C, $84.58 \%$; H, $5.28 \%$; N, $4.85 \%$.

Fabrication of COF-coated WLED. The activated 3D-TPE-COF $(\sim 10 \mathrm{mg})$ was loaded into an agate mortar and manually ground with the pestle for $10 \mathrm{~min}$ to afford a fine powder. After that, $0.5 \mathrm{~mL}$ UV curable epoxy (Norland NOA 63) was then added to the mortar, and the mixture was stirred until a homogeneous slurry was achieved. By using a simple dip-coating procedure, the mixture was uniformly dispersed on the surface of a commercially available blue LED (peaking at $\sim 450$ $\mathrm{nm}$ ), which matched well with the absorption of 3D-TPE-COF. This coating process can be easily repeated to ensure an even and continuous coating of 3DTPE-COF on the dome of the blue LED. After irradiation under UV lamp (SMC$400 \mathrm{H}$, Sunwheel Materials Co., Ltd) for $2 \mathrm{~min}$, the outer layer was then covered with a high-quality UV curable epoxy (ACW A90535-AN) which possessed a low water permeability down to $2.7 \times 10^{-4} \mathrm{~g} \mathrm{~m}^{-2}$ per day. Finally, the COF-coated 
WLED was obtained and tested at ambient conditions after curing the epoxy again for $2 \mathrm{~min}$.

\section{Data availability}

All the data supporting the findings of this study are available within the Article and its Supplementary Information, or from the corresponding author (C.W. or J. S.) upon reasonable request.

Received: 12 March 2018 Accepted: 14 November 2018 Published online: 07 December 2018

\section{References}

1. Diercks, C. S. \& Yaghi, O. M. The atom, the molecule, and the covalent organic framework. Science 355, eaal1585 (2017).

2. Das, S., Heasman, P., Ben, T. \& Qiu, S. Porous organic materials: strategic design and structure-function correlation. Chem. Rev. 117, 1515-1563 (2017).

3. Huang, N., Wang, P. \& Jiang, D. Covalent organic frameworks: a materials platform for structural and functional designs. Nat. Rev. Mater. 1, 16068 (2016).

4. Ding, S. Y. \& Wang, W. Covalent organic frameworks (COFs): from design to applications. Chem. Soc. Rev. 42, 548-568 (2013).

5. Zeng, Y., Zou, R. \& Zhao, Y. Covalent organic frameworks for $\mathrm{CO}_{2}$ capture. Adv. Mater. 28, 2855-2873 (2016).

6. Kang, Z. et al. Mixed matrix membranes (MMMs) comprising exfoliated 2D covalent organic frameworks (COFs) for efficient $\mathrm{CO}_{2}$ separation. Chem. Mater. 28, 1277-1285 (2016)

7. Han, X. et al. Chiral covalent organic frameworks with high chemical stability for heterogeneous asymmetric catalysis. J. Am. Chem. Soc. 139, 8693-8697 (2017).

8. $\mathrm{Xu}, \mathrm{H}$. S. et al. Constructing crystalline covalent organic frameworks from chiral building blocks. J. Am. Chem. Soc. 138, 11489-11492 (2016).

9. Xu, H., Gao, J. \& Jiang, D. Stable, crystalline, porous, covalent organic frameworks as a platform for chiral organocatalysts. Nat. Chem. 7, 905-912 (2015).

10. Vyas, V. S. et al. Direct optical activation of skeletal muscle fibres efficiently controls muscle contraction and attenuates denervation atrophy. Nat. Commun. 6, 8506 (2015).

11. Ding, S. Y. et al. Thioether-based fluorescent covalent organic framework for selective detection and facile removal of mercury(II). J. Am. Chem. Soc. 138, 3031-3037 (2016).

12. Dalapati, S. et al. Highly emissive covalent organic frameworks. J. Am. Chem. Soc. 138, 5797-5800 (2016).

13. Das, G. et al. Chemical sensing in two dimensional porous covalent organic nanosheets. Chem. Sci. 6, 3931-3939 (2015).

14. Jin, E. et al. Two-dimensional $\mathrm{sp}^{2}$ carbon-conjugated covalent organic frameworks. Science 357, 673-676 (2017).

15. Medina, D. D., Sick, T. \& Bein, T. Photoactive and conducting covalent organic frameworks. Adv. Energy Mater. 7, 1700387 (2017).

16. Bertrand, G. H., Michaelis, V. K., Ong, T. C., Griffin, R. G. \& Dinca, M. Thiophene-based covalent organic frameworks. Proc. Natl Acad. Sci. USA 110, 4923-4928 (2013).

17. Spitler, E. L. \& Dichtel, W. R. Lewis acid-catalysed formation of twodimensional phthalocyanine covalent organic frameworks. Nat. Chem. 2, 672-677 (2010).

18. Wang, S. et al. Exfoliation of covalent organic frameworks into few-layer redox-active nanosheets as cathode materials for lithium-ion batteries. J. Am. Chem. Soc. 139, 4258-4261 (2017).

19. Alahakoon, S. B. et al. Design principles for covalent organic frameworks in energy storage applications. ChemSusChem 10, 2116-2129 (2017).

20. Du, Y. et al. Ionic covalent organic frameworks with spiroborate linkage. Angew. Chem. Int. Ed. 55, 1737-1741 (2016).

21. Mulzer, C. R. et al. Superior charge storage and power density of a conducting polymer-modified covalent organic framework. ACS Cent. Sci. 2, 667-673 (2016).

22. Gole, B. et al. Microtubular self-assembly of covalent organic frameworks. Angew. Chem. Int. Ed. 57, 846-850 (2017).

23. Sun, Q. et al. Pore environment control and enhanced performance of enzymes infiltrated in covalent organic frameworks. J. Am. Chem. Soc. 139 2786-2794 (2017)

24. Crowe, J. W., Baldwin, L. A. \& McGrier, P. L. Luminescent covalent organic frameworks containing a homogeneous and heterogeneous distribution of dehydrobenzoannulene vertex units. J. Am. Chem. Soc. 138, 10120-10123 (2016).
25. Pang, Z.-F. et al. Construction of covalent organic frameworks bearing three different kinds of pores through the heterostructural mixed linker strategy. $J$. Am. Chem. Soc. 138, 4710-4713 (2016)

26. Zeng, Y. et al. Covalent organic frameworks formed with two types of covalent bonds based on orthogonal reactions. J. Am. Chem. Soc. 137, 1020-1023 (2015).

27. Zhou, T.-Y. et al. One-step construction of two different kinds of pores in 2D covalent organic framework. J. Am. Chem. Soc. 136, 15885-15888 (2014).

28. El-Kaderi, H. M. et al. Designed synthesis of $3 \mathrm{D}$ covalent organic frameworks. Science 316, 268-272 (2007)

29. Lin, G. et al. A pyrene-based, fluorescent three-dimensional covalent organic framework. J. Am. Chem. Soc. 138, 3302-3305 (2016).

30. Lin, G. et al. 3D porphyrin-based covalent organic frameworks. J. Am. Chem Soc. 139, 8705-8709 (2017).

31. Liu, Y. et al. Weaving of organic threads into a crystalline covalent organic framework. Science 351, 365-369 (2016).

32. Zhang, Y.-B. et al. Single-crystal structure of a covalent organic framework. J Am. Chem. Soc. 135, 16336-16339 (2013)

33. Ma, T. et al. Observation of interpenetration isomerism in covalent organic Frameworks. J. Am. Chem. Soc. 140, 6763-6766 (2018).

34. $\mathrm{Li}, \mathrm{H}$. et al. Three-dimensional covalent organic frameworks with dual linkages for bifunctional cascade catalysis. J. Am. Chem. Soc. 138, 14783-14788 (2016).

35. Baldwin, L. A., Crowe, J. W., Pyles, D. A. \& McGrier, P. L. Metalation of a mesoporous three-dimensional covalent organic framework. J. Am. Chem. Soc. 138, 15134-15137 (2016).

36. Fang, Q. et al. 3D microporous base-functionalized covalent organic frameworks for size-selective catalysis. Angew. Chem. Int. Ed. 53, 2878-2882 (2014).

37. $\mathrm{Ma}, \mathrm{H}$. et al. A $3 \mathrm{D}$ microporous covalent organic framework with exceedingly high $\mathrm{C}_{3} \mathrm{H}_{8} / \mathrm{CH}_{4}$ and $\mathrm{C}_{2}$ hydrocarbon/ $\mathrm{CH}_{4}$ selectivity. Chem. Commun. 49, 9773-9775 (2013).

38. Beaudoin, D., Maris, T. \& Wuest, J. D. Constructing monocrystalline covalent organic networks by polymerization. Nat. Chem. 5, 830-834 (2013)

39. Stewart, D. et al. Stable and ordered amide frameworks synthesised under reversible conditions which facilitate error checking. Nat. Commun. 8, 1102 (2017).

40. Wang, Y. et al. Introductory lecture: recent research progress on aggregationinduced emission. Faraday Discuss. 196, 9-30 (2017).

41. Mei, J. et al. Aggregation-induced emission: together we shine, united we soar! Chem. Rev. 115, 11718-11940 (2015).

42. Luo, J. et al. Aggregation-induced emission of 1-methyl-1,2,3,4,5pentaphenylsilole. Chem. Commun. 1740-1741 (2001)

43. Wang, C. et al. The marriage of AIE and interface engineering: convenient synthesis and enhanced photovoltaic performance. Chem. Sci. 8, 3750-3758 (2017).

44. Huang, J. et al. Highly efficient nondoped OLEDs with negligible efficiency roll-off fabricated from aggregation-induced delayed fluorescence luminogens. Angew. Chem. Int. Ed. 56, 12971-12976 (2017).

45. Kwok, R. T. K., Leung, C. W. T., Lam, J. W. Y. \& Tang, B. Z. Biosensing by luminogens with aggregation-induced emission characteristics. Chem. Soc. Rev. 44, 4228-4238 (2015).

46. Dalapati, S., Gu, C. \& Jiang, D. Luminescent porous polymers based on aggregation-induced mechanism: design, synthesis and functions. Small 12, 6513-6527 (2016)

47. Yuan, Y. \& Liu, B. Visualization of drug delivery processes using AIEgens. Chem. Sci. 8, 2537-2546 (2017)

48. Ma, L., Feng, X., Wang, S. \& Wang, B. Recent advances in AIEgen-based luminescent metal-organic frameworks and covalent organic frameworks. Mater. Chem. Front. 1, 2474-2486 (2017).

49. Shustova, N. B., McCarthy, B. D. \& Dinca, M. Turn-On fluorescence in tetraphenylethylene-based metal-organic frameworks: an alternative to aggregation-induced emission. J. Am. Chem. Soc. 133, 20126-20129 (2011).

50. Ganesan, P. et al. Tetrahedral n-type materials: efficient quenching of the excitation of p-type polymers in amorphous films. J. Am. Chem. Soc. 127, 14530-14531 (2005).

51. Luo, W. et al. A dynamic covalent imine gel as a luminescent sensor. Chem. Commun. 50, 11942-11945 (2014).

52. Wan, W., Sun, J., Su, J., Hovmöller, S. \& Zou, X. Three-dimensional rotation electron diffraction: software RED for automated data collection and data processing. J. Appl. Crystallogr. 46, 1863-1873 (2013).

53. Sheldrick, G. M. Crystal structure refinement with SHELXL. Acta Crystallogr. C. Struct. Chem. A71, 3-8 (2015).

54. Song, Z. et al. Activatable fluorescent nanoprobe with aggregation-induced emission characteristics for selective in vivo imaging of elevated peroxynitrite generation. Adv. Mater. 28, 7249-7256 (2016).

55. Gong, Q. et al. Solution processable MOF yellow phosphor with exceptionally high quantum efficiency. J. Am. Chem. Soc. 136, 16724-16727 (2014). 
56. Jang, E. et al. White-light-emitting diodes with quantum dot color converters for display backlights. Adv. Mater. 22, 3076-3080 (2010).

57. Reineke, S. et al. White organic light-emitting diodes with fluorescent tube efficiency. Nature 459, 234-238 (2009).

58. Pimputkar, S., Speck, J. S., DenBaars, S. P. \& Nakamura, S. Prospects for LED lighting. Nat. Photon. 3, 180-182 (2009).

\section{Acknowledgements}

We gratefully acknowledge financial support from the National Natural Science Foundation of China (21572170, 21772149, and 21527803), the Funds for Creative Research Groups of Hubei Province (2017CFA002), and the Beijing National Laboratory for Molecular Sciences.

\section{Author contributions}

H.D. performed the synthesis and the characterizations of 3D-TPE-COF, including NMR, PXRD, gas absorption, UV-vis, and flurenscence spectrum. G.X. performed the WLED test including the dip-coating and stability experiment. G.L., R.C., Z.P., C.Y., and B.W. assisted with the experiment. J. L. and J.S. performed cRED experiment and solve the crystal structure. C.W. and J.S. conceived the project, supervised the experiment and wrote the manuscript with the assistance of the other authors.

\section{Additional information}

Supplementary Information accompanies this paper at https://doi.org/10.1038/s41467018-07670-4.
Competing interests: The authors declare no competing interests.

Reprints and permission information is available online at http://npg.nature.com/ reprintsandpermissions/

Publisher's note: Springer Nature remains neutral with regard to jurisdictional claims in published maps and institutional affiliations.

\section{(c) (1)}

Open Access This article is licensed under a Creative Commons Attribution 4.0 International License, which permits use, sharing, adaptation, distribution and reproduction in any medium or format, as long as you give appropriate credit to the original author(s) and the source, provide a link to the Creative Commons license, and indicate if changes were made. The images or other third party material in this article are included in the article's Creative Commons license, unless indicated otherwise in a credit line to the material. If material is not included in the article's Creative Commons license and your intended use is not permitted by statutory regulation or exceeds the permitted use, you will need to obtain permission directly from the copyright holder. To view a copy of this license, visit http://creativecommons.org/ licenses/by/4.0/.

(C) The Author(s) 2018 